\title{
Safe transition from extracorporeal to intracorporeal urinary diversion following robot-assisted cystectomy: a recipe for reducing operative time, blood loss and complication rates
}

\author{
Teck Wei $\operatorname{Tan}^{1,2} \mathbb{D} \cdot$ Rajesh Nair $^{1} \cdot$ Sanad Saad $^{1} \cdot$ Ramesh Thurairaja $^{1} \cdot$ Muhammad Shamim Khan $^{1}$
}

Received: 8 May 2018 / Accepted: 19 June 2018 / Published online: 22 June 2018

(c) The Author(s) 2018

\begin{abstract}
Purpose We report the perioperative outcomes and complications after transition from extracorporeal urinary diversion (ECUD) to intracorporeal urinary diversion (ICUD) following robot-assisted radical cystectomy (RARC).

Methods Analysis of data from a prospectively maintained institutional review board-approved database of 180 patients treated with cystectomy at our institution from April 2015 to October 2017 was performed. 127 patients underwent RARC and received an ileal conduit. Only five patients received a neobladder after RARC and were excluded from analysis.

Results 68 patients had extracorporeal and 59 intracorporeal ileal conduit after RARC. There were no significant differences in patient demographics and oncological characteristics between the two groups. Of note, intracorporeal ileal conduit was associated with significantly reduced median total operative times (330 vs $375 \mathrm{~min}, p=0.019$ ), reduced median estimated blood loss (300 vs $425 \mathrm{ml}, p<0.035)$ and lower 30-day overall complication rates $(48.4 \mathrm{vs} 71.4 \%, p=0.008)$ when compared to extracorporeal diversion. However, the median length of stay, 30-90-day complication rates, mortality rates and ureteroileal anastomotic stricture rates were similar in both groups. The median operative time for RARC and intracorporeal ileal conduit was significantly shorter in the second cohort of 29 cases compared to the first 30 cases (300 vs $360 \mathrm{~min}, p=0.004)$. Other outcomes were similar in both cohorts.

Conclusion In our experience, transition from extracorporeal to intracorporeal diversion after RARC is safe, technically feasible and benefits from shorter operative times, reduced estimated blood loss, and lower 30-day overall complication rates.
\end{abstract}

Keywords Cystectomy · Postoperative complications · Robotics · Urinary bladder neoplasms · Urinary diversion

\section{Introduction}

Radical cystectomy with extended pelvic lymph node dissection is the standard treatment for muscle-invasive and highrisk non-muscle-invasive carcinoma of the bladder [1]. Over the last decade, robot-assisted radical cystectomy (RARC) is increasingly being utilized in a number of institutions to reap the benefits of minimally invasive surgery [2-5] while replicating principles of open surgery and maintaining oncological equivalence [6]. These benefits include a reduction

Teck Wei Tan

teck_wei_tan@ttsh.com.sg

1 Department of Urology, Guy's and St. Thomas' NHS Foundation Trust, Great Maze Pond, London SE1 9RT, UK

2 Department of Urology, Tan Tock Seng Hospital, 11 Jalan Tan Tock Seng, Singapore 308433, Singapore in postoperative morbidity and an earlier return to normal activities.

Most of the literature evaluating RARC, including four randomized trials comparing $\mathrm{RARC}$ with open radical cystectomy [7-10], have used extracorporeal urinary diversion (ECUD), deemed a simpler and quicker procedure than intracorporeal urinary diversion (ICUD). With increasing surgical experience, ICUD is increasingly being adopted allowing for potential benefits, including decreased fluid loss, reduced estimated blood loss (EBL), less pain, smaller incisions, a faster return of bowel function and fewer anastomotic strictures [11]. At present, there are limited studies comparing perioperative outcomes of ICUD and ECUD [12-14].

We established a RARC program in our institution in 2004 and completed the transition from ECUD to ICUD in October 2016. The objectives of this study are to report on 
the perioperative outcomes and postoperative complications following our transition to ICUD.

\section{Materials and methods}

\section{Study design}

Review of a prospectively maintained institutional review board-approved database of patients treated with cystectomy at our institution from April 2015 to October 2017 was performed. The study period was chosen to encompass the transition period from ECUD to ICUD after RARC in October 2016, with similar numbers of patients undergoing each type of diversion. During this period, 180 patients underwent cystectomy with urinary diversion at our center, of which 142 underwent RARC. 127 of these patients received an ileal conduit and were included in this study. There were only five patients who received a neobladder and they were excluded due to their small numbers. Ten patients had cutaneous ureterostomies after RARC due to the palliative intent of their surgery and were also excluded from the analysis.

Comparisons were made between ECUD and ICUD groups according to patient demographics (age, gender, body mass index (BMI), American Society of Anesthesiologists (ASA) score, history of neoadjuvant chemotherapy, and prior pelvic external beam radiotherapy), oncological characteristics ( $\mathrm{T}$ stage, $\mathrm{N}$ stage, lymph node yield, and surgical margins), perioperative data (operative time, estimated blood loss, and length of stay) and complications within 90 days of surgery (reported using the Clavien-Dindo classification).

\section{Surgical technique}

Our surgical technique for RARC with pelvic lymph node dissection has previously been described [3]. For ECUD, after cystectomy and pelvic lymph node dissection, the robot was undocked and specimen retrieved through extension of the camera port via a $5-7 \mathrm{~cm}$ peri or subumbilical midline incision. Urinary diversion with an IC was then performed extracorporeally.

Table 1 Patient demographics
For ICUD, all procedures, including bowel segment isolation and ureteroenteric anastomosis, was performed intracorporeally.

\section{Statistical analysis}

Demographic, oncological characteristics, perioperative data and complications were summarized using descriptive statistics. The statistical analyses were performed using the Mann-Whitney test for continuous variables and Chisquare test for categorical variables. All statistical analyses were performed using Stata version 15.0 (StataCorp, College Station, TX). Two-sided statistical significance was defined as $p<0.05$.

\section{Results}

\section{Patient demographics}

As described in Table 1, of the total of 127 patients, 68 underwent ECUD and 59 ICUD. There were no significant differences between the ECUD and ICUD groups with respect to median age (71 vs 69 years, $p=0.059$ ), gender $(85.7 \%$ male vs $79.0 \%$ male, $p=0.323)$, median BMI (27.0 vs 26.5, $p=0.885$ ), median ASA score (2 vs 2, $p=0.803$ ), receipt of neoadjuvant chemotherapy (13.2 vs $22.0 \%, p=0.193$ ) and history of prior pelvic external beam radiotherapy ( 16.2 vs $6.8 \%, p=0.103)$.

\section{Oncological characteristics}

There were no significant differences between the ECUD and ICUD groups with respect to the proportion of pT3/ pT4 cases ( 36.8 vs $32.2 \%, p=0.588$ ), cases of lymph node positive disease ( 16.2 vs $16.9 \%, p=0.916)$, median lymph node yield (15 vs $17, p=0.989)$ and percentage with positive surgical margins $(7.4$ vs $8.5 \%, p=0.820)$.

\begin{tabular}{lccc}
\hline & $\begin{array}{l}\text { Extracorporeal } \\
\text { diversion }(n=68)\end{array}$ & $\begin{array}{l}\text { Intracorporeal } \\
\text { diversion }(n=59)\end{array}$ & $p$ value \\
\hline Age, median (IQR) (years) & $71(66-76)$ & $69(62-74)$ & 0.059 \\
Male gender $[n(\%)]$ & $60(85.7)$ & $49(79.0)$ & 0.323 \\
Body mass index, median (IQR) $\left(\mathrm{kg} / \mathrm{m}^{2}\right)$ & $27.0(24.5-29.6)$ & $26.5(23.9-30.2)$ & 0.885 \\
American Society of Anesthesiologists (ASA) score, & $2(2-2)$ & $2(2-3)$ & 0.803 \\
$\quad$ median (IQR) & $9(13.2)$ & $13(22.0)$ & 0.193 \\
Neoadjuvant chemotherapy $[n(\%)]$ & $11(16.2)$ & $4(6.8)$ & 0.103 \\
Previous pelvic external beam radiotherapy $[n(\%)]$ & &
\end{tabular}


Table 2 Oncological characteristics

\begin{tabular}{lccc}
\hline & $\begin{array}{l}\text { Extracorporeal diver- } \\
\text { sion }(n=68)\end{array}$ & $\begin{array}{l}\text { Intracorporeal diver- } \\
\text { sion }(n=59)\end{array}$ & $p$ value \\
\hline pT2 or less $[n(\%)]$ & $43(63.2)$ & $40(67.8)$ & 0.588 \\
pT3 or pT4 $[n(\%)]$ & $25(36.8)$ & $19(32.2)$ & 0.588 \\
pN0 $[n(\%)]$ & $50(73.5)$ & $39(66.1)$ & 0.366 \\
pN1-pN3 $[n(\%)]$ & $11(16.2)$ & $10(16.9)$ & 0.916 \\
No lymph node dissection $[n(\%)]$ & $7(10.3)$ & $10(16.9)$ & 0.278 \\
Lymph node yield, median $(\mathrm{IQR})$ & $15(10-23)$ & $17(10-20)$ & 0.989 \\
Overall positive surgical margins $[n(\%)]$ & $5(7.4)$ & $5(8.5)$ & 0.820 \\
\hline
\end{tabular}

The oncological characteristics of the ECUD and ICUD groups are summarized in Table 2.

\section{Perioperative outcomes and complications}

The median total operative time for RARC and intracorporeal IC was significantly shorter than for the extracorporeal group (330 vs $375 \mathrm{~min}, p=0.019$ ). However, the median operative time for urinary diversion (i.e., formation of ileal conduit) was similar for both groups (135 min for ECUD vs $120 \mathrm{~min}$ for ICUD, $p=0.950$ ).

ICUD was associated with significantly lower EBL (300 vs $425 \mathrm{ml}, p<0.035$ ) and lower 30-day overall complication rates $(48.4$ vs $71.4 \%, p=0.008)$ compared with ECUD. However, the 30-day high-grade (Clavien-Dindo 3-5) complication rates were similar between the ECUD and ICUD groups $(10.0$ vs $8.1 \%, p=0.712)$. There were no deaths within 30 days of surgery in either groups and the median length of stay was equivalent (8 days). The 30-90day complication rate and mortality rates were similar in both groups.

As we transitioned from ECUD to ICUD just over halfway through the study period, the median follow-up duration was significantly shorter for the ICUD group (4 vs 14 months, $p<0.001)$. The ureteroileal anastomotic stricture rate was lower in the ICUD compared to the ECUD group (3.2 vs $7.4 \%, p=0.300)$, but this was not statistically significant. This may be related to the shorter duration of follow-up for the ICUD group, and also possibly related to the effects of radiotherapy, as a greater proportion of the ECUD group had previously received pelvic external beam radiotherapy (16.2 vs $6.8 \%, p=0.103)$, although this was also not statistically significant. The perioperative outcomes and complications are summarized in Table 3.

Table 4 details the learning curve effect when comparing the first cohort of 30 intracorporeal IC to the next cohort of 29 cases. We found a significantly reduced median total operative time for RARC and intracorporeal IC in the second cohort of 29 cases compared to the first 30 cases (300 vs $360 \mathrm{~min}, p=0.004)$. However, other outcomes such as EBL, length of stay, 30 and 90-day complication rates were similar in both cohorts.

Table 3 Perioperative outcomes and complications

\begin{tabular}{|c|c|c|c|}
\hline & $\begin{array}{l}\text { Extracorporeal diversion } \\
(n=68)\end{array}$ & $\begin{array}{l}\text { Intracorporeal diversion } \\
(n=59)\end{array}$ & $p$ value \\
\hline Total operative time, median (IQR) (min) & $375(313-420)$ & $330(300-368)$ & 0.019 \\
\hline Total operative time for urinary diversion, median (IQR) (min) & $135(120-180)$ & $120(120-170)$ & 0.950 \\
\hline Estimated blood loss, median (IQR) (ml) & $425(300-500)$ & $300(200-400)$ & 0.035 \\
\hline Length of stay, median (IQR) (days) & $8(7-10)$ & $8(6-11)$ & 0.166 \\
\hline Follow-up duration, median (IQR) (months) & $14(10-20)$ & $4(1-7)$ & $<0.001$ \\
\hline 30-day overall complication $[n(\%)]$ & $50(71.4)$ & $30(48.4)$ & 0.008 \\
\hline 30-day high-grade (Clavien-Dindo 3-5) complication [ $n(\%)]$ & $7(10.0)$ & $5(8.1)$ & 0.712 \\
\hline 30 days mortality $[n(\%)]$ & $0(0)$ & $0(0)$ & - \\
\hline 30-90-day overall complication $[n(\%)]$ & $10(15.2)$ & $7(16.2)$ & 0.878 \\
\hline 30-90-day high-grade (Clavien-Dindo 3-5) complication [ $n(\%)]$ & $6(9.1)$ & $5(11.6)$ & 0.645 \\
\hline 30-90-day mortality $[n(\%)]$ & $2(2.9)$ & $0(0)$ & 0.191 \\
\hline Ureteroileal anastomotic stricture $[n(\%)]$ & $5(7.4)$ & $2(3.2)$ & 0.300 \\
\hline
\end{tabular}


Table 4 Learning curve for intracorporeal urinary diversion

\begin{tabular}{|c|c|c|c|}
\hline & First 30 cases & Next 29 cases & $p$ value \\
\hline Total operative time for ileal conduit, median (IQR) (min) & $360(330-390)$ & $300(270-360)$ & 0.004 \\
\hline Estimated blood loss, median (IQR) (ml) & $325(200-438)$ & $300(200-400)$ & 0.664 \\
\hline Length of stay, median (IQR) (days) & $8(7-11)$ & $7(6-11)$ & 0.399 \\
\hline 30-day overall complication $[n(\%)]$ & $16(53.3)$ & $14(48.3)$ & 0.703 \\
\hline 30-day high-grade (Clavien-Dindo 3-5) complication $[n(\%)]$ & $3(10.0)$ & $2(6.9)$ & 0.672 \\
\hline 30 days mortality $[n(\%)]$ & $0(0)$ & $0(0)$ & - \\
\hline 30-90-day overall complication $[n(\%)]$ & $3(10.7)$ & $4(30.8)$ & 0.058 \\
\hline 30-90-day high-grade (Clavien-Dindo 3-5) complication [ $n(\%)]$ & $2(7.1)$ & $3(23.1)$ & 0.088 \\
\hline 30-90-day mortality $[n(\%)]$ & $0(0)$ & $0(0)$ & - \\
\hline
\end{tabular}

\section{Discussion}

RARC is a technically complex procedure consisting of three parts: extirpation of the bladder, pelvic lymph node dissection, and finally, urinary diversion. Patients with bladder cancer undergoing cystectomy are often elderly, chronic smokers and have multiple comorbidities. This makes the surgical procedure more challenging, and recovery, on occasion, unpredictable. ECUD is the most commonly performed technique in most centers when compared to ICUD as it is deemed quicker and technically less demanding. Ahmed et al. [13] performed an analysis of the International Robotic Cystectomy Consortium (IRCC) database, and $82 \%$ of the 935 patients received an ECUD, with only $18 \%$ of urinary diversions constructed intracorporeally.

However, with increasing surgical experience, rates of ICUD are increasing, due to its potential perceived benefits over ECUD. These include decreased fluid loss from evaporation, reduced EBL, less pain, smaller incisions and earlier return to normal bowel function [11]. Wound-related benefits may be even greater in females as the specimen can be extracted via the vagina and thus obviate the need for open extraction. There are, however, limited studies comparing perioperative outcomes of ECUD and ICUD, especially in centers transitioning from one to the other. To the best of our knowledge, our study is the largest comparing perioperative outcomes and complications from a unit transitioning between diversion techniques. We have performed RARC with ECUD since 2004 at our center [3] and transitioned to ICUD in October 2016. Late adoption of ICUD was a conscious decision to await the early published results of ICUD. It was felt that urinary diversion was a critical step in determining morbidity, functional outcomes and bowel recovery. The outcomes of our first 59 intracorporeal ileal conduit cases compared to our last 68 extracorporeal cases showed no differences in terms of patient demographics and oncological characteristics, but we found that ICUD resulted in benefits of shorter operative times, lower EBL, and 30-day complication rates.

One of the concerns with adoption of ICUD is the perceived longer operative times due to the complexity of intracorporeal bowel handling and suturing, especially early in the learning curve [15]. Having performed RARC in our unit for more than a decade, we did not experience any significant issues transitioning from ECUD to ICUD. In fact, we found that the median total operative time for RARC and intracorporeal ileal conduit to be significantly shorter (330 min compared to $375 \mathrm{~min}$ for extracorporeal, $p=0.019$ ). This is likely due to the obviation of the need for undocking of the robot for urinary diversion and the presence of experienced robotic bedside assistants. In addition, despite the training needs of our bladder cancer fellowship program, we believe that a dual console robotic system in our center ensures a safe and efficient training environment and shortens the learning curve of our fellows.

Operative times also improved with experience, with a significantly reduced median operative time for RARC and intracorporeal ileal conduit in the second cohort of 29 cases compared to the first 30 cases ( 300 vs $360 \mathrm{~min}$, $p=0.004)$. Our finding of a shorter operating time for RARC with intracorporeal ileal conduit is consistent with Novara et al.'s systemic review, which reported a mean operative time of $360 \mathrm{~min}$ for extracorporeal ileal conduit compared to $340 \mathrm{~min}$ for intracorporeal ileal conduit [16]. However, Ahmed et al.'s review of the IRCC database did not find a significant difference in the operative times between ECUD and ICUD [13]. Guru et al. described their experience transitioning from ECUD to ICUD in twenty-six patients (thirteen patients underwent ECUD and ICUD, respectively) [12]. They found that operative times were similar between the two groups. The only other published study of a unit transitioning from ECUD to ICUD was by Pyun et al. [14]. Their study consisted of 64 patients, 38 of whom underwent ECUD and 26 had ICUD. They noted ICUD resulted 
in significantly longer operative times (468 min for ECUD and $581 \mathrm{~min}$ for ICUD, $p<0.05$ ).

We also found that ICUD resulted in lower EBL compared to ECUD (300 vs $425 \mathrm{ml}, p<0.035$ ). We believe that the pneumoperitoneum and superior vision offered by utilizing the robotic approach for ICUD helped reduce blood loss by allowing more precise dissection and improved bowel division and anastomosis. The majority of the complications following radical cystectomy are usually a result of this complex reconstruction. A potential advantage of ICUD is reduced bowel handling with limited peritoneal cavity exposure to air. This is associated with reduced postoperative systemic inflammatory response and therefore improves bowel recovery $[17,18]$. In our study, we noted a lower 30-day overall complication rate of the ICUD group compared to ECUD (48.4 vs $71.4 \%, p=0.008)$. Our findings of a lower EBL and 30-day overall complication rate in favor of ICUD is mirrored in Pyun et al.'s study of their transition from ECUD to ICUD [14]. They reported a reduction in mean EBL from 265 to $148 \mathrm{ml}(p<0.001)$ and less overall complications in the ICUD group (30.8\% for ICUD and 57.9\% for ECUD, $p=0.033$ ).

Our study, however, has some limitations. First, although this study had the largest cohort to date, the sample size was still relatively small. Second, despite our patient demographics and the oncological characteristics being similar between groups, there still remains a degree of selection bias due to the non-randomized nature. In addition, with a limited follow-up of these patients, there remains a longterm uncertainty with regards to outcomes between ECUD and ICUD. We are one of the participating institutions in the Phase III prospective multi-center randomized controlled trial comparing the outcomes from intracorporeal roboticassisted radical cystectomy with open radical cystectomy (iROC trial) [19], and the results of this study will provide level 1 evidence on the outcomes of ICUD compared to open surgery.

In conclusion, our experience has shown that transitioning from ECUD to ICUD after RARC has specific benefits including shorter operative times, reduced EBL, and lower 30-day complication rates.

Author contributions T.W.T.: Project development, data collection, data analysis, and manuscript writing. R.N.: Project development and manuscript editing. S.S.: Data collection. R.T.: Project development and manuscript editing. M.S.K.: Project development and manuscript editing.

\section{Compliance with ethical standards}

Conflict of interest The authors declare no conflict of interest and do not have anything to disclose.

Ethical approval The study was approved by the institutional research committee and was performed in accordance with the ethical standards as laid down in the 1964 Declaration of Helsinki and its later amendments or comparable ethical standards.

Informed consent Informed consent was obtained from all individual participants included in the study.

Open Access This article is distributed under the terms of the Creative Commons Attribution 4.0 International License (http://creativeco mmons.org/licenses/by/4.0/), which permits unrestricted use, distribution, and reproduction in any medium, provided you give appropriate credit to the original author(s) and the source, provide a link to the Creative Commons license, and indicate if changes were made.

\section{References}

1. Alfred Witjes J, Lebret T, Compérat EM et al (2017) Updated 2016 EAU guidelines on muscle-invasive and metastatic bladder cancer. Eur Urol 71:462-475

2. Collins JW, Tyritzis S, Nyberg T et al (2013) Robot-assisted radical cystectomy: description of an evolved approach to radical cystectomy. Eur Urol 64:654-663

3. Murphy DG, Challacombe BJ, Elhage O et al (2008) Roboticassisted laparoscopic radical cystectomy with extracorporeal urinary diversion: initial experience. Eur Urol 54:570-580

4. Tan WS, Sridhar A, Goldstraw M et al (2015) Robot-assisted intracorporeal pyramid neobladder. BJU Int 116:771-779

5. Desai MM, Gill IS, De Castro Abreu AL et al (2014) Robotic intracorporeal orthotopic neobladder during radical cystectomy in 132 patients. J Urol 192:1734-1740

6. Yuh B, Wilson T, Bochner B et al (2015) Systematic review and cumulative analysis of oncologic and functional outcomes after robot-assisted radical cystectomy. Eur Urol 67:402-422

7. Parekh DJ, Messer J, Fitzgerald J et al (2013) Perioperative outcomes and oncologic efficacy from a pilot prospective randomized clinical trial of open versus robotic assisted radical cystectomy. J Urol 189:474-479

8. Khan MS, Gan C, Ahmed K et al (2016) A single-centre early phase randomised controlled three-arm trial of open, Robotic, and Laparoscopic Radical Cystectomy (CORAL). Eur Urol 69:613-621

9. Nix J, Smith A, Kurpad R et al (2010) Prospective randomized controlled trial of robotic versus open radical cystectomy for bladder cancer: perioperative and pathologic results. Eur Urol 57:196-201

10. Bochner BH, Dalbagni G, Sjoberg DD et al (2015) Comparing open radical cystectomy and robot-assisted laparoscopic radical cystectomy: a randomized clinical trial. Eur Urol 67:1042-1050

11. Wilson TG, Guru K, Rosen RC et al (2015) Best practices in robot-assisted radical cystectomy and urinary reconstruction: recommendations of the Pasadena consensus panel. Eur Urol 67:363-375

12. Guru K, Seixas-Mikelus SA, Hussain A et al (2010) Robotassisted intracorporeal ileal conduit: marionette technique and initial experience at Roswell Park Cancer Institute. Urology 76:866-871

13. Ahmed K, Khan SA, Hayn MH et al (2014) Analysis of intracorporeal compared with extracorporeal urinary diversion after robot-assisted radical cystectomy: results from the international robotic cystectomy consortium. Eur Urol 65:340-347

14. Pyun JH, Kim HK, Cho S et al (2016) Robot-Assisted Radical cystectomy with total intracorporeal urinary diversion: comparative analysis with extracorporeal. J Laparoendosc Adv Surg Tech A 26:349-356 
15. Desai MM, de Abreu ALC, Goh AC et al (2014) Robotic intracorporeal urinary diversion: technical details to improve time efficiency. J Endourol 28:1320-1327

16. Novara G, Catto JWF, Wilson T et al (2015) Systematic review and cumulative analysis of perioperative outcomes and complications after robot-assisted radical cystectomy. Eur Urol 67:376-401

17. Sammour T, Kahokehr A, Chan S et al (2010) The humoral response after laparoscopic versus open colorectal surgery: a meta-analysis. J Surg Res 164:28-37
18. Chen HH, Wexner SD, Iroatulam AJ et al (2000) Laparoscopic colectomy compares favorably with colectomy by laparotomy for reduction of postoperative ileus. Dis Colon Rectum 43:61-65

19. ClinicalTrials.gov. Trial to compare robotically assisted radical cystectomy with open radical cystectomy (iROC). https://clinicaltr ials.gov/ct2/show/NCT03049410 